vol. $37-n^{\circ} 1$ et 2 | 2021

Travail et migrations

\title{
Éditorial : La relation de travail à la croisée des politiques migratoires et économiques
}

Editorial: The Employment Relationship at the Crossroads between Migration

Policies and Economic Policies

Editorial: La relación laboral en el cruce de las políticas migratorias y económicas

\section{Camille Schmoll et Serge Weber}

\section{(Q) OpenEdition}

Journals

Édition électronique

URL : https://journals.openedition.org/remi/17992

DOI : $10.4000 /$ remi. 17992

ISSN : $1777-5418$

Éditeur

Université de Poitiers

Édition imprimée

Pagination : 7-19

ISBN : 979-10-90426-69-6

ISSN : 0765-0752

Référence électronique

Camille Schmoll et Serge Weber, «Éditorial : La relation de travail à la croisée des politiques migratoires et économiques », Revue européenne des migrations internationales [En ligne], vol. 37 - $n^{\circ} 1$ et 2 | 2021, mis en ligne le 25 novembre 2021, consulté le 15 mai 2022. URL : http:// journals.openedition.org/remi/17992 ; DOI : https://doi.org/10.4000/remi.17992 


\section{Éditorial : La relation de travail à la croisée des politiques migratoires et économiques}

\section{Camille Schmoll' et Serge Weber ${ }^{2}$}

\section{Le travail dans les études migratoires : un thème dont la présence fluctue}

Pitti (2006) insistait, dans ses travaux sur les OS algériens durant les Trente Glorieuses, sur l'importance de " tenir ensemble " les figures du travailleur et de l'immigré. Ce dossier thématique s'inscrit dans cette perspective et propose, à travers un angle résolument politique, d'explorer l'imbrication entre l'évolution des migrations internationales, les mutations des systèmes productifs et la refonte des politiques publiques (migratoires et d'asile, du marché du travail et de l'État-providence).

Si les disciplines des sciences sociales ont pu constituer des sous-champs actifs portant sur le travail (histoire sociale et ouvrière, sociologie du travail), elles ont souvent relégué à leurs périphéries la question du travail immigré, malgré l'importance et la postérité de certaines recherches sur le sujet (Sayad, 1977 ; Noiriel, 1984 et 1988 ; Green, 1985). Ce n'est pas le cas du champ interdisciplinaire des " études migratoires " qui, avec sa consolidation, a fait du travail immigré un objet d'étude central. En sociologie des migrations, la place des travailleurs immigrés est notamment venue nourrir le paradigme de réflexion post-fordiste, à travers l'étude de la segmentation du marché du travail dans les années 1970 (Piore, 1978). Ce paradigme structuraliste a inspiré, jusqu'aux années 1990, de nombreux travaux croisant segmentation nationale des marchés du travail et division internationale du travail (Harris, 1995). La critique féministe et les approches par le genre sont venues compléter et amender ces travaux, en souli-

\footnotetext{
1 Géographe, Directrice d'Études, UMR 8504 Géographie-cités/École des Hautes Études en Sciences Sociales, Campus Condorcet, Bâtiment de recherches sud, 5 cours des Humanités, 93322 Aubervilliers cedex ; camille.schmoll@ehess.fr

2 Professeur de géographie, Université Gustave Eiffel/Laboratoire Analyse comparée des pouvoirs, cité Descartes, 5 boulevard Descartes, Champs-sur-Marne, 77454 Marne-laVallée cedex 2 ; serge.weber@univ-eiffel.fr

Les coordinateurs de ce dossier tiennent à remercier Jean-François Dars et Anne Papillault qui prolongent, en mettant gracieusement à disposition la photographie de couverture, leur infatigable travail de mise en images de la recherche. Parmi leurs nombreuses contributions audiovisuelles, on pourra consulter les Histoires courtes en cliquant sur ce lien : www.llx.fr
} 
gnant le rôle crucial et historique des travailleuses immigrées dans les systèmes productifs (Morokvasic, 1984 ; Green, 1998). Les années 1990 ont ensuite vu se développer le paradigme de la globalisation : celui-ci a produit une réflexion sur les circuits mondiaux de main-d'œuvre et leur concentration dans les villes globales (Sassen, 2000), ainsi que sur la division internationale du travail reproductif et du soin (Parreñas, 2001 ; Ehrenreich et Hochschild, 2003 ; Kofman et Raghuram, 2015).

Ainsi, les études migratoires ont largement exploré la question du travail salarié. Cependant, dès la fin des années 1990, une grande partie de ces travaux $\mathrm{s}^{\prime}$ éloigne de ce thème, et se focalise sur d'autres questions - telles que la loi ou la discrimination. La catégorie du " sans-papier ", amplement étudiée dans les années 1990, n'est pas toujours explorée sous l'angle du travail et de l'exploitation, à quelques notables exceptions près (Fassin et al., 1997 ; Moulier-Boutang, 1998 ; Balibar et al., 1999). En Europe, tout se passe comme si, à la suite de la mise en place des politiques du regroupement familial et la fin de la migration officielle de travail, une partie des travaux avait suivi la pente naturelle des politiques migratoires, valorisant $\mathrm{d}^{\prime}$ autres aspects $\mathrm{du}$ processus migratoire (I'installation, l'intégration, les transformations des structures familiales, etc.) aux dépens des régimes et formes de production, consacrant ainsi la division opérée par les politiques migratoires entre migration de travail " de fait " ou " invisible " et arrêt officiel des migrations de travail (Laurens, 2020).

Ce sont d'autres figures du travail immigré qui apparaissent, dans les études migratoires, au fil des années 1990. D'abord, les employés du tertiaire, dont la part dans les marchés du travail est de plus en plus importante (Marie, 1994). Surtout, la recherche se focalise sur des figures plutôt autonomes de la migration et du travail, comme celles de l'entrepreneur transnational (Peraldi, 1999 ; Tarrius, 2000), du migrant qualifié ou des diasporas dites de la connaissance (Meyer, 2004 ; Nedelcu, 2010), des commerçants et des entrepreneurs familiaux (Ma Mung et Simon, 1990 ; Martini, 2001 ; Raulin, 2000 ; Zalc et Bruno, 2006).

Plus récemment, les études migratoires ont réinvesti la question du travail salarié, même si ce regain est encore timide et s'effectue parfois à l'ombre des approches, aujourd'hui nombreuses, attachées aux frontières et à la question humanitaire. Plusieurs recherches s'intéressent aux luttes et aux formes de mobilisation des travailleurs, aux fragmentations des statuts d'emploi et à la façon dont les politiques migratoires orchestrent la mise en mobilité des travailleurs et participent à la réorganisation du marché du travail (Jounin, 2009 ; Rea, 2013 ; Mezzadra et Neilson, 2015). Les figures du travail migrant explorées dans ces travaux sont multiples : depuis une dizaine d'années, et à la faveur du regain de leurs mobilisations sur les lieux d'emploi, les travailleurs sans papiers s'imposent dans l'espace public et dans la recherche (Barron et al., 2014). D'autres travaux explorent la part des travailleurs détachés - plus d'1 demimillion en France en 2017 - dans la diffusion du précariat immigré (Castel, 2007). On assiste plus généralement, dans ces récents travaux sur les travailleurs immigrés, au retour d'une critique matérialiste, attentive à la façon dont les mobilités migratoires de travail s'articulent au capital en contexte post-fordiste ou néolibéral et au caractère utilitariste de l'appel à la main-d'œuvre (Morice et Potot, 2010 ; Filhol, 2013 ; McDowell, 2013 ; Zeneidi, 2013). 


\section{Quand politique migratoire et politique économique forgent les figures du travail migrant}

Dans les travaux actuels, capitalisme avancé et intensification des frontières (Balibar, 1994) s'imbriquent pour forger de nouvelles figures du travail migrant. C'est à cette réflexion, articulant politique migratoire et politique économique, que ce double dossier souhaite s'attacher en abordant l'articulation des statuts migratoires, légaux et professionnels à la fois sous l'angle juridique et socioéconomique : le croisement des rapports de domination au sein du travail migrant, l'impact des dispositifs de sécurisation des frontières et de contrôle des entrées sur les mondes du travail, ainsi que les formes de résistance et d'opposition à l'assignation, à l'exploitation, à l'assujettissement des travailleurs migrants ou des travailleurs impliqués dans la gestion des flux. Ces différents angles d'approche permettront de montrer que la question du travail ne peut être réduite aux mécanismes d'un marché désarrimé du ou de la politique. Elle demeure aujourd'hui révélatrice des effets politiques de la flexibilisation des conditions de travail et du déni de reconnaissance auquel sont confrontés les travailleuses et les travailleurs. L'ubérisation du marché du travail, les nouvelles formes de travail non rémunéré (bénévolat, stages), le télétravail, le travail à domicile ou les horaires atypiques sont tout autant d'aspects d'une tendance de politique économique qui dépasse la question du travail migrant tout en inscrivant celle-ci au cœur de ses développements. Cette politique introduit de nombreuses divisions et hiérarchisations, entre travailleurs européens ou extraeuropéens par exemple. Elle est également genrée : les systèmes de welfare, par exemple, reposent en grande partie sur le travail des femmes migrantes - des assistantes maternelles, nounous, auxiliaires de vie, aidessoignantes, infirmières - qui occupent une place toujours plus importante au sein de ces nouvelles figures du travail immigré, au sein desquelles la dimension reproductive, affective et émotionnelle est centrale.

\section{Nouvelles frontières dans le travail, nouvelles frontières du travail}

L'ancrage, de même que la conduite de la trajectoire migratoire, peut se faire par le travail à défaut d'un accès à une résidence stable, malgré la précarité et, parfois, l'illégalité des statuts ; ces mondes sociaux du travail croisent légalité et illégalité, formalité et informalité, travail sous contrat et hors contrat, ethnicisation des rapports de travail, à des degrés divers, selon qu'elle concerne les travailleurs détachés européens ou les travailleurs précaires non européens. Cette " porte entrouverte ", qui légitime à la fois les entraves à la reconnaissance juridique des nouveaux venus et la banalisation du travail précaire, flexible et aux marges du droit, crée des hiérarchies, plus ou moins stables, que la " promesse d'embauche " (Jounin, 2009) ou la " promesse d'une régularisation " (Di Cecco, 2020) parviennent à rendre tolérables.

Le désengagement des États amène les entreprises, dans des secteurs comme le bâtiment et la restauration, à prendre de fait en main la fonction de régulation des marchés du travail (par l'usage abondant de la sous-traitance par exemple) et la frontière se fait de plus en plus insidieuse, multilocalisée (Mezzadra et Neilson, 2015), à échelle infranationale (au niveau des négociations interpersonnelles) ou mondiale. Les familles, dans une certaine mesure, se font également 
employeuses, contribuant ainsi à troubler la limite entre rapport de travail et sphère de l'intime, comme l'illustre l'article de Maurizio Artero, Minke Hajer et Maurizio Ambrosini dans ce dossier.

À l'échelle internationale, la mobilité des travailleurs peine à trouver un cadre de régulation. Pourtant, ce cadre existe, avec la Convention internationale pour les droits des travailleurs migrants. II est révélateur que les pays signataires qui ont ratifié la convention soient presque tous des pays d'émigration, essentiellement situés dans le Sud global (De Guchteneire et Pécoud, 2010). Les pays du Nord, force est de le constater, n'en veulent pas. De fait, les fonctionnements du marché du travail aujourd'hui diffèrent sensiblement de ceux de l'après-guerre et des Trente Glorieuses : la régulation étatique et les agendas politiques ont évolué, privilégiant par exemple les accords de réadmission avec les pays tiers aux accords de main-d'œuvre.

Les dispositifs de sécurisation des frontières et de contrôle des entrées se sont tellement intensifiés depuis les années 1990 qu'ils deviennent des mondes économiques à part entière (Rodier, 2012) ; ils ont mis en danger et sous pression le droit d'asile et la protection des personnes, tant pour les réfugiés eux-mêmes que pour les professionnels de l'accueil et de l'examen des requêtes. La question du droit de travailler pour les demandeurs d'asile est en ce sens une question cruciale et ambivalente, qui ne résout pas les difficultés que rencontrent les réfugiés statutaires à mettre en œuvre des trajectoires professionnelles correspondant à leurs qualifications (Tcholakova, 2014). En parallèle, les restrictions des voies d'entrée légales et de régularisation renforcent la tension sur la demande d'asile. Ainsi, les mondes de l'asile eux-mêmes sont réinterrogés à l'aune de la problématique du travail, comme le montre Simone Di Cecco dans ce dossier. Les lieux du contrôle et du filtrage des entrées connectent la thématique du travail à celle de la logique humanitaire, notamment à travers la réflexion sur la mise au travail des demandeurs d'asile et la montée des métiers liés à la gestion des flux, entre humanitaire et sécuritaire (Fassin, 2001). Ces emplois, publics ou associatifs le plus souvent, font face à une pénurie de moyens et à des injonctions contradictoires qui interrogent le sens et les conditions du travail de I'accueil (Kobelinsky, 2012), ils donnent lieu à de nouvelles économies morales de l'accueil. On voit donc émerger plusieurs " mondes du travail " : d'une part, le travail des personnes qui interviennent dans les instances d'accueil, d'accompagnement et de bénévolat, qui contribue à brouiller de plus en plus la frontière entre travail rémunéré et travail gratuit dans les professions qui composent le "Tiers Secteur " (santé, assistance juridique et éducative, etc.). D'autre part, les dispositifs de contrôle, d'identification et de sélection des nouveaux entrants mobilisent des actifs nombreux (police aux frontières, garde-côtes, professionnels du transport ou de la sécurité) et contribuent pour leur part à démultiplier les rapports de sous-traitance et de contractualisation par appels d'offres. Ensuite, la libre circulation, instaurée de manière graduelle au sein des États européens, met en concurrence différents régimes de mobilité et produit de nouvelles hiérarchisations au sein même de la main-d'œuvre immigrée. Enfin, on assiste, dans plusieurs pays d'Europe, à une " réfugiéisation " du marché du travail (Dines et Rigo, 2015) : les demandeurs d'asile, en particulier, sont de plus en plus amenés à travailler du fait de nouvelles régulations de la procédure d'accès aux droits, qui ont une visée tout aussi morale qu'économique. 
Mais hors d'Europe, la situation du marché du travail peut se trouver à l'opposé de la réfugéisation décrite plus haut. Ainsi, l'article d'Assaf Dahdah dans ce dossier intervient-il en utile contrepoint, en nous livrant une généalogie des figures du déplacé et du réfugié au Liban, en particulier des Palestiniens et des Syriens. II met en évidence la tension entre l'intégration au sein du marché du travail des travailleurs immigrés "ethnicisés " arrivés par le biais de la kafala, système d'embauche et d'encadrement des travailleurs étrangers, et la mise au ban des exilés ressortissants arabes, aux marges de la ville. Ils montrent combien les politiques de désarabisation et de recrutement ethnique agissent de concert dans la production d'une " classe servile ", qui se distingue justement des déplacés et réfugiés.

\section{Travailleurs et travailleuses, entre exploitations et mobilisations}

Le travail de qui, au juste ? Qu'il s'agisse des sans-papiers et/ou des demandeurs d'asile, les étrangers récemment arrivés, dans des conditions rendues de plus en plus difficiles par des politiques restrictives du contrôle des entrées, nous amènent à aborder à nouveaux frais ces figures du travail migrant. Qu'on les nomme " migrants " ou " exilés ", les personnes soumises à l'attente, à l'incertitude et à la mobilité sous contrainte (Michalon, 2012) dans le long " couloir des exilés " (Agier, 2011) et les méandres de la recherche d'un statut stable (Fogel, 2019) se trouvent particulièrement vulnérables dans leur entrée sur le marché du travail. Leur accès au travail nous amène également à comprendre les mécanismes qui tolèrent ou intègrent, selon les configurations, les " passagers clandestins ". II nous porte à interroger la tension entre déracinement, errance et ancrage dans des situations locales bien spécifiques : qu'on pense aux femmes travailleuses sans papiers ballotées au gré des hébergements que proposent les opérateurs du 115 en France et cependant employées à domicile (Le Bars, 2018), aux sans-papiers en errance résidentielle employés dans l'entretien des couloirs du métro chaque matin, aux travailleurs agricoles étrangers qui enchaînent les saisons et les campements temporaires (Perrotta et Sacchetto, 2014), etc.

Les formes de résistance ou d'opposition à l'assignation, à l'exploitation, à l'assujettissement des travailleurs migrants ou des travailleurs impliqués dans la gestion des flux sont multiples et font l'objet de recherches émergentes, à la fois sur le plan des mobilisations collectives (grèves, occupations, manifestations) et sur celui des résistances moins visibles, infrapolitiques, qui révèlent les injustices associées à la condition de migrant et témoignent bien d'une cristallisation des rapports sociaux dans un monde soi-disant ouvert (Brücker et al., 2019). Ces multiples formes d'action à la marge montrent que, loin d'une dépolitisation, la question du travail demeure éminemment politique. Les résistances sont également celles des femmes ${ }^{3}$.

Les contributions rassemblées dans ce dossier mettent à jour l'articulation entre la reconfiguration des régimes du travail et celle des régimes migratoires, faisant du travail immigré un enjeu central pour repenser l'émergence d'une nouvelle forme de rapport social de production, qui repose à la fois sur le recul du droit du travail et le recul des droits humains.

3 Le genre des mobilisations avait été interrogé par des historiennes comme Linda Guerry (2009a et 2009b) et des sociologues comme Catherine Quiminal ou Claudie Lessellier. 


\section{Présentation des articles}

Les articles qui composent ce dossier s'articulent autour de cinq thématiques majeures.

\section{Aux marges du camp : entre exclusion juridique et utilitarisme économique}

La première concerne la façon dont les indésirables peuvent se muer en opportunité économique. L'orientation des exilés vers des camps ou des centres de rétention joue comme un étendard politique dans les rapports entre gouvernement de l'accueil et opinion publique. Pour autant, les personnes qui y expérimentent des conditions de vie très précaires et l'attente d'un statut juridique représentent une opportunité économique pour l'emploi dans des secteurs où la pénurie de main-d'œuvre s'accommode de la vulnérabilité et du stigmate pour proposer des conditions de travail dures.

Rocio Negrete Peña montre comment le demi-million de réfugiés républicains espagnols entrés en France entre 1936 et 1940 a été rapidement érigé à la fois comme problème politique et comme opportunité économique. L'utilitarisme économique a servi de justification face à la diffusion dans l'opinion publique de représentations de l'étranger indésirable qui ont accompagné leur accueil. Nombre d'employeurs, notamment exploitants agricoles, ont eu recours à ces exilés comme main-d'œuvre, contraignant les travailleurs agricoles français à accepter les mêmes conditions. La mise en concurrence des travailleurs est un phénomène tout sauf nouveau, qui fait jouer les intérêts immédiats du patronat et ses relations avec le mouvement ouvrier. Le contexte était particulier : entre les effets sur l'emploi de la crise de 1929, la montée d'une extrême droite protectionniste et xénophobe et le mouvement ouvrier, ces arrivées ont accentué des contradictions et des frictions qui traversaient la société. Ces divergences traversaient le monde syndical lui-même, entre ceux qui voulaient éviter la concurrence des travailleurs étrangers et ceux qui ont pris leur défense, tout en convergeant vers la défense de tous les travailleurs opprimés. De l'autre côté, le nationalisme et le racisme de l'extrême droite entendaient " assainir " le marché du travail en contrôlant strictement le recours aux travailleurs étrangers, alors que le patronat y voyait au contraire son intérêt. Les lois successives de " protection de la main-d'œuvre française " ont contribué à cantonner les exilés espagnols aux secteurs touchés par la pénurie de main-d'œuvre et les femmes au travail domestique, arrimant strictement le permis de séjour à cette assignation. Ces exilés ont été vus comme de "faux réfugiés", comme un "contingent d'indésirables ". Jusqu'à ce que, la guerre approchant, leur mise au travail comme les reconnaisse " éléments utiles pour la nation".

Le travail de Lucas Puygrenier a été mené dans un contexte a priori peu comparable avec le cas français des années 1930. Ses observations ont été menées au temps présent à Malte, un contexte insulaire et de petite taille. II est très frappant, mais finalement peu surprenant, de lire des analyses de faits qui semblent se répéter au cours de l'histoire et dans des contextes géographiques différents avec la même symétrie. L'arrivée d'un nombre important d'exilés sur l'île et la mise en place des structures pour les orienter sont vues comme des contraintes jugées excessives, alimentant des discours et des actions de rejet. 
La découverte de l'opportunité d'une présence sur le territoire d'étrangers en situation précaire et $d^{\prime}$ incertitude alimente non seulement le recrutement des étrangers en attente dans des conditions précaires, mais aussi la dynamique de croissance économique et de performance de certains secteurs, celui de la construction notamment. Ces deux articles apportent de précieux éclairages sur l'articulation entre camp, incertitude juridique et utilitarisme économique. Ces recherches confirment l'immuabilité du " lien étroit entre des lieux d'exclusion des exilés et leur "inclusion" au titre de main-d'œuvre ".

\section{Fragilité institutionnelle, ethnicisation et assignations professionnelles}

La deuxième thématique de ce dossier concerne la relation entre la régulation étatique ou régionale des migrations et la production de frontières ethniques et institutionnelles. Ces articles portent sur l'organisation par les provinces ou les États de secteurs spécifiques de recrutement de la main-d'œuvre immigrée, les barrières symboliques et/ou réelles instituées à l'entrée d'autres secteurs, et les frontières ethniques produites au quotidien, dans le monde du travail et jusque dans l'intimité des familles.

Leyla Sall explore un monde relativement peu connu et peu documenté, surtout pour les lecteurs hexagonaux : celui d'une province francophone du Canada, I'Acadie du Nouveau-Brunswick, qui mène une politique d'attraction de nouveaux habitants francophones. Ceux-ci sont confrontés à un autre type de contradiction : autant la promotion de l'immigration de pays francophones et la défense par cette province d'une reconnaissance institutionnelle à la fois de minorité et de francophonie permettent à de nouveaux arrivants d'amorcer leur parcours, en particulier pour leurs études, autant l'accès aux métiers où I'usage du français est le plus recherché, l'enseignement et la santé, se heurte à des barrières implicites et subjectives qui constituent une forme inattendue de discrimination. Cette recherche auprès de jeunes immigrés francophones met en lumière deux résultats principaux : I'un est l'ambivalence du statut de minorité linguistique protégée, confrontée à des enjeux de peuplement et de maintien d'une culture identitaire et communautaire qui peine à concilier la nécessaire ouverture à l'altérité ; I'autre concerne la valorisation du plurilinguisme. En effet, alors qu'ils sont refoulés par un plafond de verre dans les emplois en français, les immigrants francophones sont valorisés dans des secteurs d'emploi anglophones où la maîtrise d'autres langues est appréciée, avec un utilitarisme affiché. Étonnamment, ce sont donc les secteurs non francophones qui contribuent à la dynamique d'intégration de ces migrants francophones.

Assaf Dahdah éclaire la notion de prolétariat des services en analysant le système libanais de l'emploi précaire, reposant déjà sur l'assignation identitaire et l'ethnicisation dans sa segmentation, offrant des conditions d'emploi spécifiques selon l'origine nationale. Le contexte libanais est celui d'une forte immigration historique de réfugiés, provenant de pays essentiellement de pays arabes (palestiniens d'abord, syriens ensuite), à laquelle vient s'ajouter une immigration de pays non arabes, asiatiques ou africains. Cette surabondance d'offres de travail stimule l'exacerbation de la mise en concurrence entre travailleurs, en particulier avec l'arrivée massive des réfugiés syriens depuis le début de la guerre. Les Syriens non déclarés, corvéables à merci, de même que les 
travailleuses d'Afrique subsaharienne, d'Asie du Sud ou des Philippines viennent alimenter cette armée de réserve. Leur point commun est la dépendance multidimensionnelle aux employeurs en raison du système juridique de la kafala, mais ils sont confrontés à des stéréotypes différents : à la différence des exilés des pays arabes voisins qui sont associés à des a priori d'ordre politique, les personnes venues de plus loin, participant à la globalisation de la domesticité ont été vues comme une opportunité de " désarabiser " et "d'asiatiser " ce segment du travail subalterne. Les assignations à la fois ethniques et professionnelles jouent donc à plein. Mais la crise économique qui frappe le Liban depuis 2019 a une conséquence inattendue : acceptant d'être payés en livres libanaises, les exilés syriens gardent leur emploi pendant que les autres, préférant être payés en dollars, sont licenciés, endettés et piégés par la crise.

\section{La dimension morale des relations d'emploi en situation de précarité juridique}

La troisième direction de recherche apporte des éclairages sur les économies morales de l'emploi des étrangers en situation précaire.

En 2013, 2,3 millions de familles italiennes délèguent le soin aux plus âgés à des travailleurs, et surtout des travailleuses du care employées à domicile. L'article de Maurizio Artero, Minke Hajer et Maurizio Ambrosini décrit ce que les régimes migratoires font aux relations intimes à partir de l'analyse de la cohabitation entre employées du soin (badanti) et personnes âgées et vulnérables. L'article met en lumière l'ambivalence de la relation triangulaire qui lie les travailleuses immigrées, les personnes dont elles s'occupent, et les " managers de soin " le plus souvent des femmes, membres de la famille - qui sont en charge de gérer la relation de travail. L'ambivalence qui caractérise l'économie morale de ces relations quasi familiales est mise en lumière, entre affection, réciprocité et exploitation, violence parfois.

C'est précisément la légitimité de la présence au travail des étrangers en situation irrégulière qu'étudient Sébastien Chauvin, Stefan Le Courant et Lucie Tourette. Ils proposent dans leur texte d'unir leurs recherches auprès de travailleurs étrangers en situation irrégulière et nous en offrent une analyse croisée. La manière dont se négocient la légitimité, la valeur et le mérite dans ce moment biographique de l'irrégularité éclaire une économie morale. Leur analyse confirme qu'il est important de prendre en compte le travail dans l'accès à la régularisation du séjour, ce que les recherches récentes polarisées par les urgences humanitaires laissent au second plan. La primauté du travail est encore une fois confirmée : même si un certain nombre de régularisations apparaissent pour un motif familial, c'est l'ensemble du dossier qui est pris en compte et en particulier l'insertion dans l'emploi. Étant à la fois en situation irrégulière et expulsables, les travailleurs sans papiers sont pris dans la complexité des rapports économiques et moraux qui les lient à leurs employeurs. Ils ne peuvent que difficilement se retourner contre leurs employeurs et, inversement, ceux-ci estiment que leurs employés auraient une dette morale puisqu'ils ont employé des personnes a priori inemployables. Alors même que les régularisations consécutives aux grèves récentes des sans-papiers en France ont révélé la diversité des situations de travail et l'articulation entre performance économique et citoyenneté, la question de ce " salariat bridé " n'est pas pour autant réglée 
une fois qu'on a obtenu un titre de séjour : il faut pérenniser la relation de travail, faire face aux discriminations et préparer la demande de renouvellement du permis de séjour.

\section{Bénévolat et preuves d'employabilité : la gratuitisation du travail des exilés}

La quatrième direction de recherche développée dans ce dossier concerne, en articulation avec la réflexion sur les économies morales, la place des exilés dans la production d'un monde du travail lié à l'asile. Les deux situations de bénévolat étudiées témoignent de ce que Simonet (2018) avait nommé la " gratuitisation du travail ».

Maureen Clappe nous dévoile un monde bien particulier, celui des interprètes bénévoles qui, en attente d'obtenir un statut, acceptent des missions de travail afin de consolider un dossier d'attestations ou un " passeport bénévole ", qu'ils espèrent pouvoir valoriser afin d'obtenir des papiers. Ces emplois, conditions d'une possible régularisation, appelés hope labors, conjuguent les ambivalences. Ces "bénéficiaires au travail " sont à la fois public accueilli par les associations et personnel indispensable. Eux-mêmes anciens demandeurs d'asile, reconnus, déboutés, voire encore en procédure de demande d'asile, avec ou sans titre de séjour, ils se rendent utiles et s'acquittent de la dette d'avoir été aidés, tout en accumulant des preuves de civisme et de citoyenneté. Leur situation confirme que les associations sont un monde du travail spécifique, où la générosité, la garantie de " bonne conduite ", le mérite et la preuve d'employabilité peuvent générer une relation de type clientélaire. C'est un travail non salarié, mais dédommagé, qui peut être un pis-aller faute de mieux autant qu'une perspective de valoriser un dossier. C'est ainsi qu'on passe du bénévolat d'agrément au bénévolat institué.

Simone Di Cecco met en lumière une autre contradiction : les exilés qui sont en procédure de demande d'asile sont pris en charge dans des centres de statuts variés en Italie. L'allocation et la prise en charge dans ces centres d'hébergement entrent en compétition avec l'emploi. En effet, rien n'empêche les demandeurs d'asile d'exercer une activité rémunérée sur le marché de l'emploi, à partir de deux mois après leur demande d'asile. Mais l'hébergement des demandeurs d'asile est conditionné à un plafond de ressources, très bas. Ce qui fait que les demandeurs d'asile qui ont l'opportunité de trouver un emploi mieux payé sont contraints à quitter le dispositif d'hébergement et que les autres y renoncent pour une situation d'attente précaire hautement inconfortable. De cette contradiction naît une situation pour le moins paradoxale : pour valoriser, aux yeux d'une opinion publique de plus en plus méfiante vis-à-vis des nouveaux arrivants, l'image des exilés comme socialement et économiquement utiles, il leur est proposé d'occuper une activité de type professionnel, qui soit d'intérêt général, peu ou pas rémunérée, peu valorisante et indifférente à leurs qualifications. L'auteur dévoile alors la manière dont ces personnes composent avec les contraintes, avec leurs frustrations et leurs aspirations.

\section{Organisation collective, réappropriation et légitimité}

Enfin, une dernière direction de recherche porte sur les mobilisations, résistances et formes d'organisation des travailleuses et travailleurs étrangers, qui réussissent, par des voies alternatives, à institutionnaliser des processus de reconnaissance. 
Emeline Zougbédé étudie le processus de routinisation des mobilisations de travailleurs sans papiers. Son étude de plusieurs grèves menées dans les années 2010, dans le sillage de celles de 2008-2009, a pour objet l'articulation entre situation de travail et situation administrative. L'évolution législative, depuis les années 2000, a introduit l'admission exceptionnelle au séjour par le travail. C'est alors bien comme grèves du travail que les mobilisations collectives des travailleurs sans papiers peuvent se construire et participer à la reconnaissance de la place des sans-papiers dans le monde de l'emploi. Dans ce cas également, la dépendance vis-à-vis des employeurs est forte, en particulier pour les fiches de salaire et les promesses d'embauche qu'il faut fournir dans la procédure de régularisation. Cette dépendance renseigne sur la précarité vue, au-delà de l'instabilité des situations de travail en intérim et en sous-traitance, comme la prolongation de situations d'incertitude et la nécessité d'accumuler des preuves de performance économique. Ces grèves ont permis la réappropriation d'une identité sociale et économique ainsi que d'un registre de dispositions professionnelles et organisationnelles. L'articulation avec un syndicat, la CGT, a permis l'appropriation de " routines conflictuelles " qui sont entendues par les interlocuteurs : ces grèves du travail s'institutionnalisent.

Le dernier article de ce dossier résulte de deux enquêtes ethnographiques menées en Suisse, par Christina Mittmasser et Isabella Stingl, auprès de réfugiés et de travailleurs sociaux impliqués dans des programmes spécifiques de travail, et auprès d'entrepreneurs migrants. L'analyse conjointe éclaire le fonctionnement d'une organisation originale, fondée et animée par des migrants, qui contraste avec les programmes institutionnels mis en place pour l'emploi des exilés. Les principales actions tentent de faire contrepoids à trois tendances qui génèrent de fortes frustrations et manquent, au prix d'un gâchis évitable, les opportunités d'une insertion professionnelle plus cohérente. D'abord la déqualification et la dévaluation des compétences généralement observées sur le marché de l'emploi, ensuite, les catégories de pensée dominantes qui contribuent, en associant des stéréotypes d'altérité à des processus d'exclusion, à dévaloriser la place les migrants dans le monde du travail, enfin, la réduction des exilés à de simples travailleurs subalternes. Cette initiative autonome permet de légitimer un autre regard sur la place des étrangers dans l'emploi. 


\section{Références bibliographiques}

Agier Michel (2011) Le couloir des exilés. Être étranger dans un monde commun, Vulaines-sur-Seine, Éditions du Croquant.

Balibar Étienne, Chemillier-Gendreau Monique, Costa-Lascoux Jacqueline et Terray Emmanuel (1999) Sans papiers. L'archaïsme fatal, Paris, La Découverte.

Balibar Étienne (1994), Qu'est-ce qu'une frọtière ?, in Marie-Claire CalozTschopp, Axel Clevenot et Maria-Pia Tschopp Éds., Asile, Violence, Exclusion en Europe, Genève, Section des Sciences de l'Éducation, Université de Genève et Groupe de Genève, pp. 335-343.

Barron Pierre, Bory Anne, Chauvin Sébastien, Jounin Nicolas et Tourette Lucie (2014) Derrière le sans-papiers, le travailleur: Genèse et usages de la catégorie de " travailleurs sans papiers " en France, Genèses, 94 (1), pp. 114-139.

Brücker Pauline, Veron Daniel et Vertongen Youri Lou (2019) Des mouvements de sans-papiers à la " crise " des réfugiés : évolution des catégories d'action et enjeux théoriques, Critique internationale, 3 (84), pp. 9-21.

Castel Robert (2007) Au-delà du salariat ou ên deçà de l'emploi ? L'institutionnalisation du précariat, in Serge Paugam Éd., Repenser la solidarité. L'apport des sciences sociales, Paris, PUF, pp. 415-433.

De Guchteneire Paul et Pécoud Antoine (2010) Les obstacles à la ratification de la Convention des Nations Unies sur la protection des droits des travailleurs migrants, Droit et société, 75 (2), pp. 431-451.

Di Cecco Simone (2020) En Italie, le sale boulot de I'intégration, Plein droit, $126(3)$, pp. 32-35.

Dines Nick and Rigo Enrica (2015) Postcolonial citizenships and the refugeeization of the workforce: migrant agricultural labour in the Italian Mezzogiorno, in Sandra Ponzanesi and Guido Colpani Eds., Postcolonial transitions in Europe: Contexts, practices and politics, Lanham MD, Rowman and Littlefield, pp. 153-174.

Ehrenreich Barbara and Hochschild Arlie Russell (Eds.) (2003) Global woman: nannies, maids and sex workers in the new economy, New York, Metropolitan Books.

Fassin Didier (2001) Quand le corps fait loi. La raison humanitaire dans les procédures de régularisation des étrangers, Sciences sociales et santé, 19 (4), pp. 5-34.

Fassin Didier, Morice Alain et Quiminal Catherine (Dirs.) (1997) Les lois de I'inhospitalité. Les politiques de l'immigration à l'épreuve des sans-papiers, Paris, La Découverte.

Filhol Romain (2013) Les travailleurs agricoles migrants en Italie du Sud. Entre incompréhension, instrumentalisation et solidarités locales, Hommes et migrations, 1301, pp. 139-147.

Fogel Frédérique (2019) Parenté sans papiers, La Roche-sur-Yon, Éditions Dépaysage.

Green Nancy (1998) Du Sentier à la Septième Avenue. La confection et les immigrés, Paris-New York, 1880-1980, Paris, Seuil.

Green Nancy (1985) Les travailleurs immigrés juifs à la Belle Époque : le " Pletzl » de Paris, Paris, Fayard. 
Guerry Linda (2009a) Femmes et genre dans I'histoire de l'immigration. Naissance et cheminement d'un sujet de recherche, Genre \& Histoire, [en ligne] consulté le 13/10/2021. URL : http://journals.openedition.org/genrehistoire/808

Guerry Linda (2009b) Les grèves oubliées des immigrantes à Marseille, Plein droit, 82 (3) pp. 36-40.

Harris Nigel (1995) The New Untouchables: Immigration and the New World Worker, New York, I. B. Tauris.

Jounin Nicolas (2009) Chantier interdit au public. Enquête parmi les travailleurs du bâtiment, Paris, La Découverte.

Kobelinsky Carolina (2012) " II n'y a que I'expulsion à I'horizon " : le dilemme des travailleurs sociaux des centres d'accueil pour demandeurs d'asile en France, in Michel Agier Dir., Réfugiés, sinistrés, sans papiers. Politiques de l'exception, Paris, Téraèdre, pp. 24-35.

Kofman Eleonore and Raghuram Parvati (2015) Gendered Migrations and Global Social Reproduction. Migration Diaspora and Citizenship, Basingstoke, Palgrave.

Laurens Sylvain (2020) Dérogations et " machine de I'exception ». L'après 1974 dans les couloirs des ministères, Hommes et Migrations, 1330, pp. 13-30.

Le Bars Joanne (2018) Accéder à un espace à soi. Contraintes de genre, luttes de classement et résistances en situation administrative précaire, Annales de géographie, 720 (2), pp. 169-191.

Ma Mung Emmanuel et Simon Gildas (1990) Commerçants maghrébins et asiatiques en France, Paris, Masson.

Marie Claude-Valentin (1994) L'immigration en France dans les années quatrevingt-dix : nouvelle donne pour l'emploi et nouveaux enjeux de société, Sociologie du Travail, 36 (2), pp. 143-163.

Martini Manuela (2001) Famille et organisation du travail dans les PME de la banlieue parisienne au XXe siècle, Cahier des Annales de Normandie, 31, pp. 93-10.

Martini Manuela et Rygiel Philippe (Dirs.) (2009) Genre et travail migrant. Mondes atlantiques, XIXe-XXe sieccles, Paris, Publibook.

McDowell Linda (2013) Working Lives: Gender, Migration and Employment in Britain, 1945-2007, London, Wiley \& Sons.

Meyer Jean-Baptiste (2004) Les diasporas de la connaissance : atout inédit de la compétitivité du Sud, Revue internationale et stratégique, 55, pp. 69-76.

Mezzadra Sandro and Neilson Brett (2015) Border as Method, or, the Multiplication of Labor, Durham, Duke University Press.

Michalon Bénédicte (2012) La mobilité au service de l'enfermement ? Les centres de rétention pour étrangers en Roumanie, Géographie et cultures, 81, pp. 91-110.

Morice Alain et Potot Swanie (Dirs.) (2010) De l'ouvrier immigré au travailleur sans papiers. Les étrangers dans la modernisation du salariat, Paris, Karthala.

Morokvasic Mirjana (1984) Birds of Passage are also Women..., International Migration Review, 18 (4), pp. 886-907.

Moulier-Boutang Yann (1998) De l'esclavage au salariat. Économie historique du salariat bridé, Paris, Presses Universitaires de France. 
Nedelcu Mihaela (2010) (Re)penser le transnationalisme et l'intégration à l'ère du numérique. Vers un tournant cosmopolitique dans l'étude des migrations internationales ?, Revue Européenne des Migrations Internationales, 26 (2), pp. 33-55.

Noiriel Gérard (1988) Le creuset français. Histoire de l'immigration (XIXe-XXe siècle), Paris, Seuil.

Noiriel Gérard (1984) Longwy. Immigrés et prolétaires (1880-1980), Paris, Presses Universitaires de France.

Parreñas Rhacel (2001) Servants of globalization. Women, migration and domestic work, Stanford, Stanford University Press.

Peraldi Michel (1999) Marseille : réseaux migrants transfrontaliers, place marchande et économie de bazar, Cultures \& Conflits, 33-34, pp. 51-67.

Perrotta Domenico and Sacchetto Devi (2014) Migrant Farmworkers in Southern Italy: Ghettoes, Caporalato and Collective Action, Workers of the World, 1 (5), pp. 75-98.

Piore Michael (1978) Dualism in the Labour Market, Revue économique, 19 (1), pp. 26-37.

Pitti Laure (2006) La main d'œuvre algérienne dans l'industrie automobile (19451962) ou les oubliés de I'histoire, Hommes et migrations, 1263, pp. 47-57.

Raulin Anne (2000) L'ethnique est quotidien. Diasporas, marchés et cultures métropolitaines, Paris, L'Harmattan.

Rea Andrea (2013) Les nouvelles figures du travailleur immigré : fragmentation des statuts d'emploi et européanisation des migrations, Revue Européenne des Migrations Internationales, 29 (2), pp. 15-35.

Rodier Claire (2012) Xénophobie Business, Paris, La Découverte.

Sassen Saskia (2000) Women's burden. Counter-geographies of globalization and the feminization of survival, Journal of International Affairs, 53 (2), pp. 503-524.

Sayad Abdelmalek (1977) Les trois " âges " de l'émigration algérienne en France, Actes de la recherche en sciences sociales, 15, pp. 59-79.

Simonet Maud (2018) Travail gratuit : la nouvelle exploitation ?, Paris, Textuel.

Tarrius Alain (2000) Les nouveaux cosmopolitismes : mobilités, identités, territoires, LaTour d'Aigues, Éditions de l'Aube.

Tcholakova Albena (2014) La relation de service comme rapport de reconnaissance : I'exemple de l'accompagnement des réfugiés vers le travail, Travailler, 32 , pp. 99-129.

Terray Emmanuel (2008) L'État nation vu par les sans-papiers, Actuel Marx, $44(2)$, pp. 41-52.

Zalc Claire et Bruno Anne-Sophie (Éds.) (2006) Petites entreprises et petits entrepreneurs étrangers en France (XIXe-XXe siècle). Actes des journées d'études des 23 et 24 octobre 2003, Paris, Publibook.

Zeneidi Djemila (2013) Femmes/fraises. Import/export, Paris, Presses Universitaires de France. 